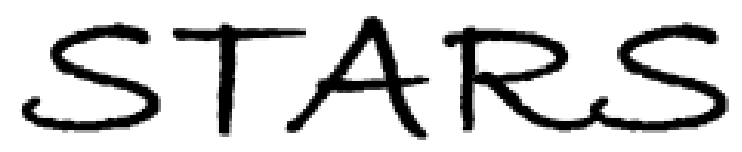

University of Central Florida

STARS

$1-1-2013$

\title{
Impact of charge impurities on transport properties of graphene nanoribbons
}

Christian W. Smith

University of Central Florida

Jyoti Katoch

University of Central Florida

Masa Ishigami

University of Central Florida

Find similar works at: https://stars.library.ucf.edu/facultybib2010

University of Central Florida Libraries http://library.ucf.edu

This Article is brought to you for free and open access by the Faculty Bibliography at STARS. It has been accepted for inclusion in Faculty Bibliography 2010 s by an authorized administrator of STARS. For more information, please contact STARS@ucf.edu.

\section{Recommended Citation}

Smith, Christian W.; Katoch, Jyoti; and Ishigami, Masa, "Impact of charge impurities on transport properties of graphene nanoribbons" (2013). Faculty Bibliography 2010s. 2573.

https://stars.library.ucf.edu/facultybib2010/2573

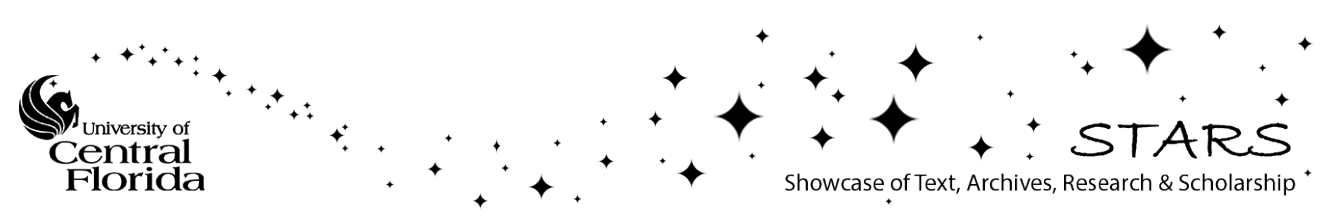




\section{Impact of charge impurities on transport properties of graphene nanoribbons}

Cite as: Appl. Phys. Lett. 102, 133502 (2013); https://doi.org/10.1063/1.4799675

Submitted: 09 November 2012 . Accepted: 21 March 2013. Published Online: 01 April 2013

Christian W. Smith, Jyoti Katoch, and Masa Ishigami
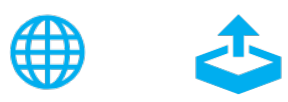

View Online

Export Citation

\section{ARTICLES YOU MAY BE INTERESTED IN}

Charged impurity-induced scatterings in chemical vapor deposited graphene

Journal of Applied Physics 114, 233703 (2013); https://doi.org/10.1063/1.4852435

Localized charge carriers in graphene nanodevices

Applied Physics Reviews 2, 031301 (2015); https://doi.org/10.1063/1.4926448

Electron mobility calculation for graphene on substrates

Journal of Applied Physics 116, 083703 (2014); https://doi.org/10.1063/1.4893650

\section{Applied Physics Reviews} Now accepting original research 


\title{
Impact of charge impurities on transport properties of graphene nanoribbons
}

\author{
Christian W. Smith, Jyoti Katoch, and Masa Ishigami \\ Department of Physics and Nanoscience Technology Center, University of Central Florida, Orlando, \\ Florida 32816-2385, USA
}

(Received 9 November 2012; accepted 21 March 2013; published online 1 April 2013)

\begin{abstract}
Previous experimental studies have shown qualitative dependence of transport property of graphene nanoribbons on external charged impurities. We have measured transport properties of a graphene nanoribbon at increasing coverage of charged impurities in an ultra high vacuum environment. We discovered an exact relationship between the source-drain and gate gaps at increasing charged impurity density. In addition, we found that graphene nanoribbons have different electronic screening as compared to bulk graphene. Our study paves the way for controlling transport property of nanoribbons using extrinsic impurities. (c) 2013 American Institute of Physics. [http://dx.doi.org/10.1063/1.4799675]
\end{abstract}

Graphene sheets, individual layers of graphite, exhibit unique physical properties. Its utility in electronics is defined by extraordinary field effect mobility at room temperature. ${ }^{1}$ However, graphene is a semimetal, which possesses zero electronic bandgap. As such, the utility of graphene in electronics is limited to devices that do not require high on-off ratio. There are several methods to engineer bandgaps in graphene. $^{2-4}$ The technique, which is most compatible with the existing microelectronic processing technologies, is to narrow graphene sheets into nanoribbons. A previous theoretical study ${ }^{5}$ has predicted electronic bandgaps of nanoribbons with perfect, disorder-free edges to be a function of their widths, orientations with respect to the lattice, and edge terminations.

Transport properties of nanoribbons, ${ }^{2,6}$ created using electron beam lithography, are characterized by an insulating behavior at finite levels of source-drain bias voltage, $\mathrm{V}_{\mathrm{sd}}$, and at certain gate voltage, $\mathrm{V}_{\text {gate }}$. These are referred to as the source-drain and gate gaps. The gate gap is also referred to as the transport gap. These gaps do not correlate to the theoretically predicted electronic bandgaps, and the substratebound charged impurities are suspected to determine the observed gaps. 6,7 Such discrepancies can be attributed to nanoribbons being composed of coupled quantum dots, which are defined by width variations imparted by the resolution limit in the lithography process and potential fluctuations induced by charge impurities. The gate gap is then a function of the magnitude of the potential fluctuation, and the source-drain gap is a function of the size of the dots and the strength of the coupling between the dots. This charged impurity picture for nanoribbons has not been tested directly as a function of the density of charged impurities on nanoribbons. Elucidating the impact of charged impurities is important as controlling the relevant gap is critical to the utility of nanoribbons.

We deposited increasing densities of adsorbed charged impurities on otherwise atomically clean graphene nanoribbons to elucidate the impact of charged impurities on the energy gaps. We have found an exact relationship between the source-drain and gate gaps at increasing charged impurity density, which is consistent with the charged impurity picture. Our study paves the way for predicting and controlling transport properties of nanoribbons.

Graphene sheets, used for our experiments, were obtained from Kish graphite by mechanical exfoliation on degenerately doped silicon wafer, which has an oxide thickness of $280 \mathrm{~nm}$. Doped silicon acts as a back gate during transport measurements. Before making graphene, these wafers are cleaned in piranha solution $\left(\mathrm{H}_{2} \mathrm{SO}_{4}: \mathrm{H}_{2} \mathrm{O}_{2}=1: 3\right)$ and sonicated in clean, deionized water. Graphene sheets are identified using the analysis of the $2 \mathrm{D}$ peak by Raman
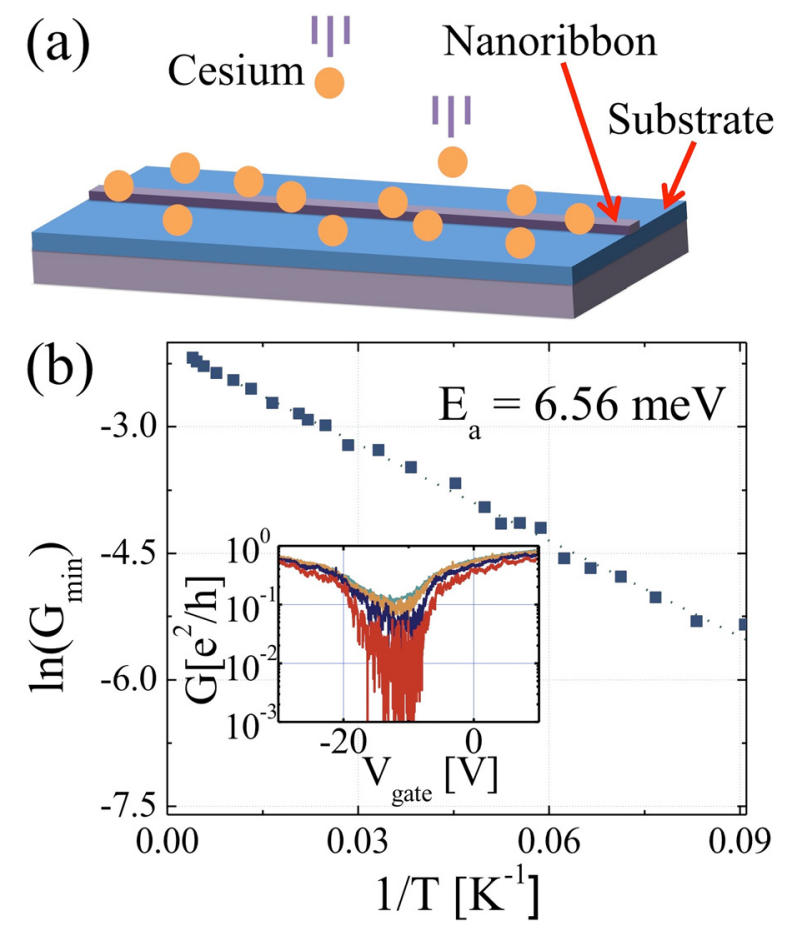

FIG. 1. (a) Schematics of the experiment. (b) Arrhenius plot for the nanoribbon prior depositing any cesium. The plot was created from data obtained by measuring the temperature dependence of the minimum conductance. The minimum conductance is determined by measuring the transport property as a function of $\mathrm{V}_{\text {gate }}$. The inset shows the measurements performed at 270, 95, 30 , and $11 \mathrm{~K}$. 


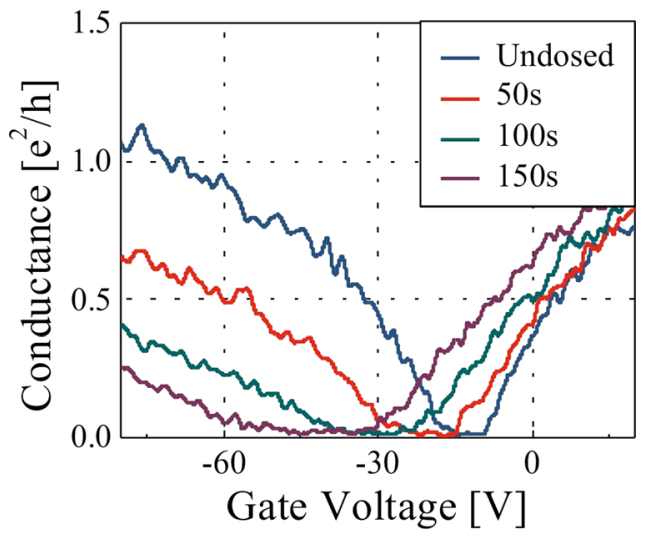

FIG. 2. Gate dependent conductance of the nanoribbon at increasing coverage of nanoribbon.

spectroscopy. ${ }^{8}$ Electron beam lithography using the standard electron beam resist ${ }^{9}$ is used to define contacts, which are metallized using gold with a chromium sticking layer. Etch mask patterns for nanoribbons are also defined using electron beam lithography, and unwanted graphene is removed using low-energy reactive ion etching. Measurements using a $40 \mathrm{~nm}$ wide and $900 \mathrm{~nm}$ long nanoribbon are discussed in our paper. Prior to measurements, graphene nanoribbons are cleaned down to atomic scale by annealing in an argon/ hydrogen atmosphere at $300^{\circ} \mathrm{C}$ for $3 \mathrm{~h}$ at atmospheric pressure. ${ }^{10}$

Clean devices are introduced to a helium flow cryostat operated in an ultra high vacuum (UHV) environment. Devices are annealed at $495 \mathrm{~K}$ for 12 to $20 \mathrm{~h}$ to remove any surface adsorbates before experiments are performed at 11 $\mathrm{K}$. During the experiment, the device is exposed successively to a constant cesium flux, produced by a cesium getter, for 10 -s intervals. The dose rate is estimated to be $1.74 \times 10^{13}$ atoms $/\left(\mathrm{s} \mathrm{m}^{2}\right)$ or 0.09 cesium/(s $\left.\mu \mathrm{m}\right)$ by measuring the partial pressure of cesium using a residual gas analyzer ${ }^{11}$ at the sample location immediately following the experiment. ${ }^{12}$ At the maximum coverage reached in our experiment, the surface density of cesium is $2.59 \times 10^{15}$ atoms $/ \mathrm{m}^{2}$ with the distance between cesium being $20 \mathrm{~nm}$. At such dilute coverage, cesium is expected to be randomly distributed on the nanoribbon and the substrate as shown in Figure 1(a) according to a previous tunneling microscopy experiment performed for potassium on graphite. ${ }^{13}$ Transport properties of the nanoribbon are measured after each dosage interval.
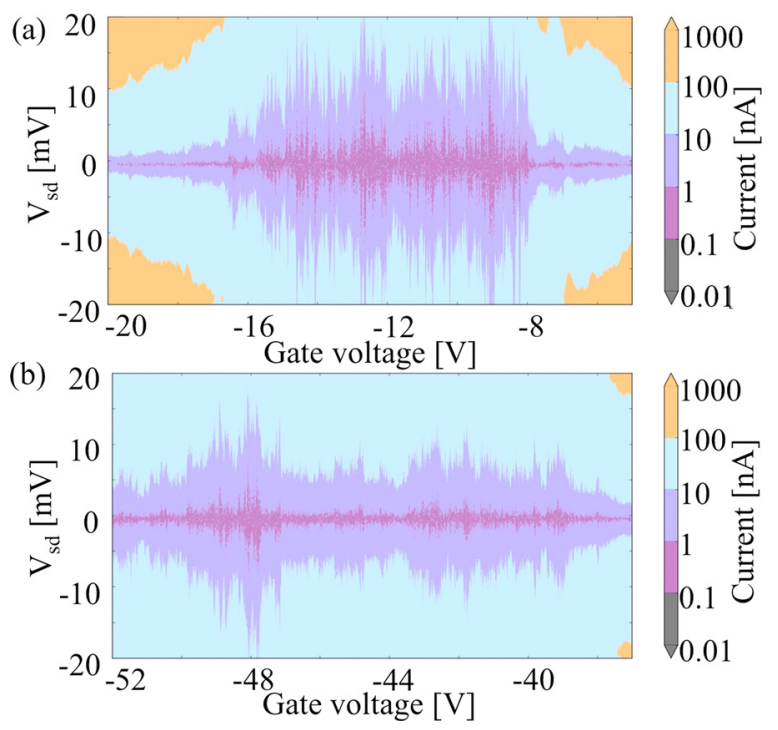

FIG. 4. (a) Current measured as a function of bias and gate voltage for the annealed ribbon. (b) Measured current of the ribbon after saturation. (The same y-scale is used for both (a) and (b).)

Figure 1(b) shows the thermally activated behavior of the nanoribbon before depositing cesium. Temperature dependent conductivity measurements enable us to calculate the activation gap of our nanoribbons. The activation energy for the particular nanoribbon is found to be $6.56 \mathrm{meV}$, which is comparable to what has been seen in nanoribbons with similar widths. ${ }^{14}$

The field-effect mobility of graphene is observed to be $360 \mathrm{~cm}^{2} /(\mathrm{V} \mathrm{s})$ for holes and $410 \mathrm{~cm}^{2} /(\mathrm{V} \mathrm{s})$ for electrons prior to dosing with cesium. Figure 2 shows the gate dependent conductivity of the nanoribbon at increasing coverage of cesium. Cesium reduces the field effect mobility, shifts the charge neutrality point to more negative value, and affects the source-drain and gate gaps. We now discuss the impact of cesium in detail.

Adsorbed cesium decreases the field effect mobility. Figure 3(a) shows the inverse electron and hole mobilities, $1 / \mu_{\mathrm{e}}$ and $1 / \mu_{\mathrm{h}} .1 / \mu_{\mathrm{e}}$ shows a sub-linear dependence while $1 / \mu_{\mathrm{h}}$ shows a super-linear dependence on the charge impurity density. Linear dependence on the charge impurity density is expected from considering diffusive transport in graphene, ${ }^{15}$ and our data may indicate that the Boltzmann transport theory alone is unable to describe charged-impurity scattering in nanoribbons.
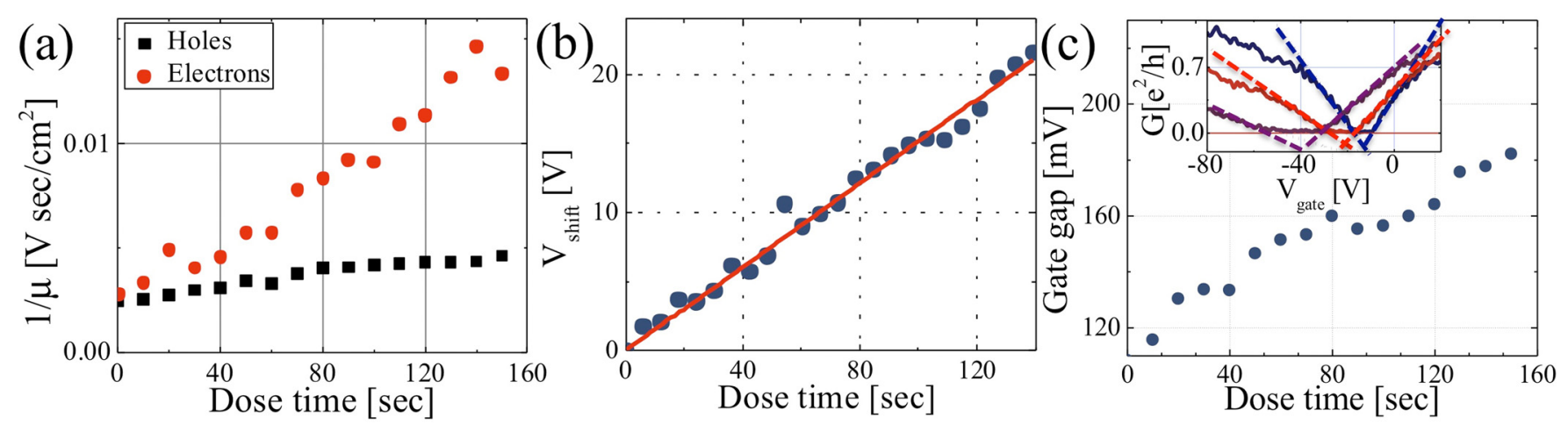

FIG. 3. (a) Inverse mobility, $1 / \mu$, as a function of the charge impurity density. (b) Charge neutrality point shift as a function of the charge impurity density. (c) Gate gap as a function of the charge impurity density. The inset shows how the gate gap was determined at various dosage times. 

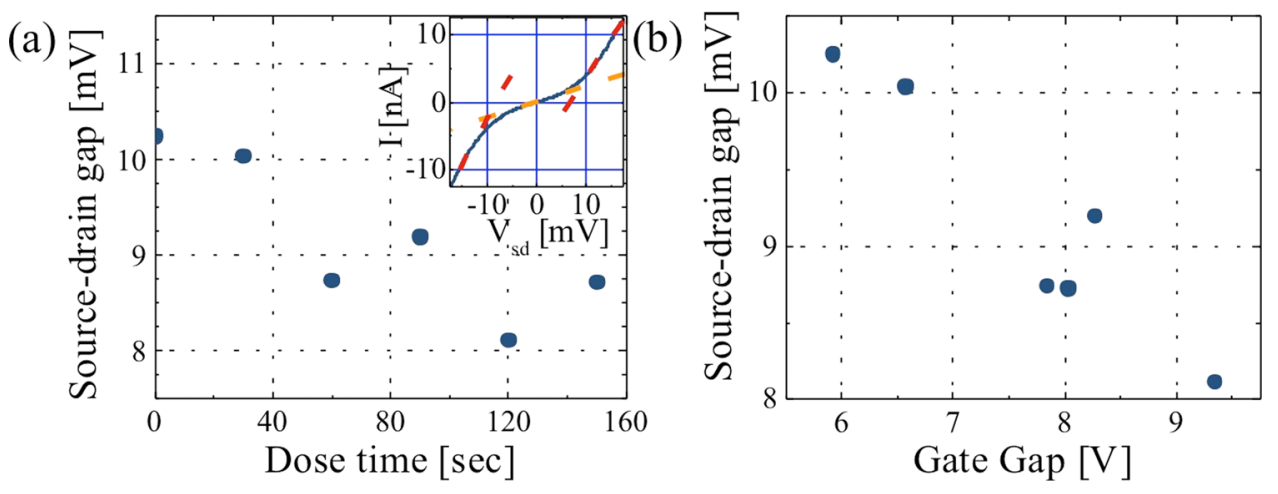

FIG. 5. (a) The effect of added impurities on the source-drain gap. The inset shows how the source-drain gap was determined by measuring the current, I, as a function of the source-drain voltage, $\mathrm{V}_{\text {sd. }}$ (b) The relationship between the two gaps.
A linear fit to the low carrier density regime and extrapolation to zero conductance determines the turn-on gate voltages for electrons and holes. The charge neutrality point is defined to be exactly half way between these turn-on voltages and cesium shifts this neutrality point to more negative values. We define $\mathrm{V}_{\text {shift }}$ to be the shift of the neutrality point induced by cesium. Figure 3(b) shows the dependence of $\mathrm{V}_{\text {shift }}$ on the charge impurity density. The relationship is given by $\mathrm{V}_{\text {shift }}=(0.15 \pm 0.002) \times \mathrm{t}$, where $\mathrm{t}$ is the dose time in seconds. The observed linear-dependence is different from bulk graphene ${ }^{16}$ in which $\mathrm{V}_{\text {shift }}$ has a superlinear power law dependence on the charge impurity density, showing that charge screening is different for nanoribbons as compared to bulk graphene. A calculation, using the geometric capacitance formula with a phenomenological reduction factor ${ }^{17}$ of 2 , indicates that 0.29 electrons/(s $\mu \mathrm{m})$ are added to the nanoribbon. This value is within the same order of magnitude as expected from our partial pressure measurement assuming 0.6-0.8 electrons transferred from each adsorbed cesium atom. ${ }^{18}$

The difference between the electron and hole turn-on gate voltages is the gate gap. ${ }^{6}$ Figure 3 (c) show that the gate gap increases as the dose time is increased. Such increase is expected in the framework set by the previous discussion of the possible influence of charged impurities on nanoribbons. Potential fluctuations caused by charged impurities increase the insulating region.

Figure 4 shows the device characteristics as a function of source-drain bias for a wide range of gate voltages within the region of suppressed conductance before dosing and after dosing for $150 \mathrm{~s}$. The gapped region shows a clear increase in the gate gap, but an overall decrease in the source-drain gap at increasing coverage of cesium. Source-drain gaps are determined at the gate voltage corresponding to the largest amplitude of conductance suppression. As shown in Figure 5 (a), the measured source-drain gap for the nanoribbon decreases as the dose time is increased. Figure 5(b) shows the measured value of the source-drain and gate gap for various impurity concentrations. The relationship is given by $\Delta_{\text {sd }}$ $=\left(-0.64 \times 10^{-3} \Delta_{\mathrm{g}}+14.1\right) \mathrm{meV}$, where $\Delta_{\mathrm{sd}}$ is the sourcedrain gap and $\Delta_{\mathrm{g}}$ is the gate gap in volts. Such linear, monotonic relationship is different from the previous measurements of the source-drain gap as a function of widths ${ }^{6}$ and thermal treatments. ${ }^{7}$

The observed behavior of the source-drain gap is consistent with the charged impurity picture in which the source-drain gap is defined by the size of the smallest dot. The charging energy of a dot is inversely proportional to the size of the dot. ${ }^{14}$ Added adsorbed cesium is situated only $2.8 \AA$ away from graphene. ${ }^{18}$ Thus, the spatial extent of the adsorbate-induced Coloumb potential on graphene can exceed the potential due to the substrate-bound charged impurities, which are typically assumed to be more than $1 \mathrm{~nm}$ away. ${ }^{15}$ As such, the size of the smallest dot can be expanded at increasing charge impurity density, increasing the source-drain gap.

We have measured the impact of adsorbed charged impurity on graphene nanoribbons. A monotonic relationship between the gate and source-drain gaps, which can be explained in the charged impurity picture, ${ }^{7}$ and different electronic screening property of nanoribbons as compared to bulk graphene have been observed. Our study paves the way for controlling the transport properties of nanoribbons using extrinsic impurities.

This work is based upon research supported by the National Science Foundation under Grant No. 0955625. The authors would like to thank Eduardo Mucciolo and Shaffique Adam for helpful discussions and Enrique del Barco for sharing instruments in his Lab. C.W.S. fabricated devices and performed the experiments, J.K. assisted with the measurements, and M.I. conceived the experiments and helped to interpret the data.

${ }^{1}$ A. K. M. Newaz, Y. S. Puzyrev, B. Wang, S. T. Pantelides, and K. I. Bolotin, Nat. Commun. 3, 734 (2012).

${ }^{2}$ M. Y. Han, B. Özyilmaz, Y. Zhang, and P. Kim, Phys. Rev. Lett. 98(20), 206805 (2007).

${ }^{3}$ Z. Chen, Y.-M. Lin, M. J. Rooks, and P. Avouris, Physica E 40(2), 228-232 (2007).

${ }^{4}$ K. Todd, H.-T. Chou, S. Amasha, and D. Goldhaber-Gordon, Nano Lett. 9(1), 416-421 (2009).

${ }^{5}$ K. Nakada, M. Fujita, G. Dresselhaus, and M. S. Dresselhaus, Phys. Rev. B 54(24), 17954 (1996).

${ }^{6}$ F. Molitor, A. Jacobsen, C. Stampfer, J. Güttinger, T. Ihn, and K. Ensslin, Phys. Rev. B 79(7), 075426 (2009).

${ }^{7}$ P. Gallagher, K. Todd, and D. Goldhaber-Gordon, Phys. Rev. B 81(11), 115409 (2010).

${ }^{8}$ A. C. Ferrari, J. C. Meyer, V. Scardaci, C. Casiraghi, M. Lazzeri, F. Mauri, S. Piscanec, D. Jiang, K. S. Novoselov, S. Roth, and A. K. Geim, Phys. Rev. Lett. 97(18), 187401 (2006).

${ }^{9}$ Poly methyl methacrylate (PMMA) / methyl methacrylate (MMA) bilayer resist.

${ }^{10}$ M. Ishigami, J. H. Chen, W. G. Cullen, M. S. Fuhrer, and E. D. Williams, Nano Lett. 7(6), 1643-1648 (2007).

${ }^{11}$ Residual gas analyzer, RGA 200, from Stanford Research Systems was used for these measurements.

${ }^{12}$ Cesium partial pressure of $1.7 \mathrm{e}-11$ Torr was measured. We calculate the flux using cesium temperature of $900 \mathrm{~K}$ (SAES getters).

${ }^{13}$ J. Renard, M. B. Lundeberg, J. A. Folk, and Y. Pennec, Phys. Rev. Lett. 106(15), 156101 (2011). 
${ }^{14}$ C. Stampfer, S. Fringes, J. Guttinger, F. Molitor, C. Volk, B. Terres, J. Dauber, S. Engels, S. Schnez, A. Jacobsen, S. Droscher, T. Ihn, and K. Ensslin, Fron. Phys. 6(3), 271 (2011).

${ }^{15}$ S. Adam, E. H. Hwang, V. M. Galitski, and S. D. Sarma, Proc. Natl. Acad. Sci. U.S.A. 104(47), 18392-18397 (2007).
${ }^{16}$ J. H. Chen, C. Jang, S. Adam, M. S. Fuhrer, E. D. Williams, and M. Ishigami, Nat. Phys. 4(5), 377-381 (2008).

${ }^{17}$ Y.-M. Lin, V. Perebeinos, Z. Chen, and P. Avouris, Phys. Rev. B 78, 161409(R) (2008).

${ }^{18}$ M. Caragiu and S. Finberg, J. Phys. Condens. Matter 17, R995 (2005). 